\title{
NEW ORE-TYPE CONDITIONS FOR $H$-LINKED GRAPHS
}

\author{
MICHAEL FERRARA ${ }^{1}$, RONALD GOULD ${ }^{2}$, MICHAEL JACOBSON $^{3}$, FLORIAN PFENDER $^{4}$, \\ JEFFREY POWELL ${ }^{5}$, THOR WHALEN ${ }^{6}$
}

\begin{abstract}
For a fixed (multi)graph $H$, a graph $G$ is $H$-linked if any injection $f: V(H) \rightarrow V(G)$ can be extended to an $H$-subdivision in $G$. The notion of an $H$-linked graph encompasses several familiar graph classes, including $k$-linked, $k$ ordered and $k$-connected graphs. In this paper, we give two sharp Ore-type degree sum conditions that assure a graph $G$ is $H$-linked for arbitrary $H$. These results extend and refine several previous results on $H$-linked, $k$-linked and $k$-ordered graphs.
\end{abstract}

All graphs in this paper are finite. For notation not defined here we refer the reader to [1]. If $X \subseteq V(G)$ is a vertex set, we will often just write $X$ for the induced subgraph $G[X]$ if the context is clear. Given an integer-valued graph parameter $p$ and a graph property $\mathcal{P}$, the $p$-threshold for $\mathcal{P}$ is the minimum $k=k(n)$ such that any graph $G$ of order $n$ with $p(G) \geq k$ has property $\mathcal{P}$. We will frequently consider $p$-thresholds restricted to specific graph classes, such as sufficiently large graphs, or graphs with a prescribed number of edges.

Let $\delta(G)$ and $\Delta(G)$ denote the minimum and maximum degree of $G$, respectively, and let $\sigma_{2}(G)$ denote the minimum degree sum of nonadjacent vertices in $G$. Throughout the paper, we will often refer to $\sigma_{2}$ conditions as Ore-type conditions in light of Ore's classical theorem on hamiltonian graphs. We will also let $n_{i}(G)$ be the number of vertices of degree $i$ in $G$.

A graph $G$ is $k$-linked if for any ordered subset of $2 k$ vertices $S=\left\{s_{1}, t_{1}, \ldots, s_{k}, t_{k}\right\}$ there exist disjoint paths $P_{1}, \ldots, P_{k}$ such that for each $i, P_{i}$ is an $s_{i}-t_{i}$ path. We will refer to this collection of paths as an $S$-linkage in $G$. We also say that $G$ is $k$-ordered if for any list of $k$ vertices $v_{1}, \ldots, v_{k}$ in $G$, there exists a cycle that visits these vertices in the given order.

For a fixed graph $H$, a graph $G$ is $H$-linked if any injection $f: V(H) \rightarrow V(G)$ can be extended to an $H$-subdivision in $G$. We refer to the injection $f$ as an $H$-linkage

1991 Mathematics Subject Classification. 05C38, 05 C83.

Key words and phrases. $H$-linked Graph, $k$-linked Graph, Degree Conditions.

${ }^{1}$ University of Colorado Denver, Denver CO, michael.ferrara@ucdenver.edu

${ }^{2}$ Emory University, Atlanta GA, rg@mathcs . emory .edu

${ }^{3}$ University of Colorado Denver, Denver CO, michael.jacobson@ucdenver.edu

${ }^{4}$ Universität Rostock, Rostock, Germany, Florian.Pfender@uni-Rostock.de

${ }^{5}$ Samford University, Birmingham, AL, jspowel1@samford.edu

${ }^{6}$ Methodic Solutions, Inc., thorwhalen@gmail.com 
problem (in $G$ ). The notion of an $H$-linked graph generalizes those of $k$-linked, $k$ ordered and $k$-connected graphs, as $G$ is $k K_{2}$-linked if and only if $G$ is $k$-linked, $G$ is $C_{k}$-linked if and only if $G$ is $k$-ordered and $G$ is $k$-connected if and only if $G$ is $K_{1, k}$-linked.

\section{Degree Conditions for $H$-Linked Graphs}

Kawarabayashi, Kostochka and Yu [8] determined sharp minimum degree and degree sum conditions for a graph $G$ of order at least $2 k$ to be $k$-linked.

Theorem 1. Let $G$ be a graph on $n \geq 2 k$ vertices. If

or

$$
\delta(G) \geq \begin{cases}\frac{n+2 k-3}{2}, & \text { if } n \geq 4 k-1 \\ \frac{n+5 k-5}{3}, & \text { if } 3 k \leq n \leq 4 k-2 \\ n-1, & \text { if } 2 k \leq n \leq 3 k-1\end{cases}
$$

$$
\sigma_{2}(G) \geq \begin{cases}n+2 k-3, & \text { if } n \geq 4 k-1 \\ \frac{2(n+5 k)}{3}-3, & \text { if } 3 k \leq n \leq 4 k-2 \\ 2 n-3, & \text { if } 2 k \leq n \leq 3 k-1\end{cases}
$$

then $G$ is $k$-linked. These bounds are best possible.

For sufficiently large graphs, the relevant portion of these conditions were obtained independently in [6]. Sharp minimum degree and degree sum conditions for $k$-ordered graphs were determined in [2] and [9], respectively.

Theorem 2. Let $G$ be a graph of order $n$ and $k \geq 2$ be an integer. If

(a) $n \geq 11 k-3$ and $\delta(G) \geq\left\lceil\frac{n}{2}\right\rceil+\left\lfloor\frac{k}{2}\right\rfloor-1$, or

(b) $n \geq 53 k^{2}$ and $\sigma_{2}(G) \geq n+\left\lceil\frac{3 k-9}{2}\right\rceil$,

then $G$ is k-ordered.

Turning our attention the the broader class of $H$-linked graphs, minimum degree conditions that assure a graph $G$ is $H$-linked for arbitrary connected $H$ were first given in [3] and [10]. These were subsequently strengthened in [5] to include arbitrary multigraphs $H$, thereby extending Theorem 1. Similar conditions concerned with finding (strong) $H$-immersions in a graph $G$ appear in [4]. In order to discuss these results, we must first introduce a useful parameter.

For a (multi-)graph $H$, let

$$
b(H)=\max _{\substack{A \cup B \cup C=V(H) \\ V(H) \neq C}}|E(A, B)|+|C| .
$$

As every graph $G$ has a bipartite subgraph with at least half of the edges in $G$, $b(H) \geq|E(H)| / 2$. When $H$ is connected, it is straightforward to see that we may choose $C$ to be empty in any optimal partition, so that $b(H)$ is equal to the maximum 
number of edges in a bipartite subgraph of $H$. As was noted in [4] and [5], when $H$ is disconnected, $b(H)$ depends not only on the maximum size of a bipartite subgraph of $H$, but also on the number of components of $H$ without even cycles.

The following result of Gould, Kostochka and $\mathrm{Yu}$ gives the $\delta$-threshold for $H$ linkedness and also represents the current best bound on the necessary order of the target graph $G$.

Theorem 3. Let $H$ be a (multi-)graph with $c(H)$ components that do not contain even cycles and $G$ be a graph of order $n \geq 9.5(|E(H)|+c(H)+1)$. If

$$
\delta(G) \geq \frac{1}{2}(n+b(H)-2),
$$

then $G$ is H-linked. This result is sharp.

Kostochka and $\mathrm{Yu}[11]$ gave Ore-type conditions, dependent on $k$, implying that a graph $G$ is $H$-linked for every graph $H$ with $k$ edges.

Theorem 4. Let $G$ be a graph of order $n$ and let $H$ be a simple graph with $k$ edges and minimum degree at least two. If

$$
\sigma_{2}(G) \geq\left\{\begin{array}{cl}
\left\lceil n+\frac{3 k-9}{2}\right\rceil & n>2.5 k-5.5 \\
\left\lceil n+\frac{3 k-8}{2}\right\rceil & 2 k \leq n \leq 2.5 k-5.5 \\
2 n-3 & k \leq n \leq 2.5 k-1,
\end{array}\right.
$$

then $G$ is H-linked.

In light of Theorem 2, one interesting consequence of Theorem 4 is that amongst those graphs $H$ with $k$ edges, $C_{k}$ has the largest $\sigma_{2}$-threshold for $H$-linkedness when $n$ is sufficiently large.

The goal of this paper is to refine Theorem 4 by giving sharp Ore-type conditions that assure a graph $G$ is $H$-linked for an arbitrarily chosen $H$. We note here that the $\sigma_{2}$-threshold for $H$-linkedness is not, in general, twice the minimum degree given in Theorem 3, as Theorem 2 demonstrates that this is not the case for $H=C_{k}$ when $n$ is sufficiently large. Our first result demonstrates that twice the minimum degree in Theorem 3 does suffice if we add only a mild minimum degree condition to $G$.

Theorem 5. Let $H$ be a multigraph and $G$ be a graph with $|G| \geq 20|E(H)|+n_{0}(H)$. If

$$
\begin{aligned}
\delta(G) & \geq 4|E(H)|+n_{0}(H), \text { and } \\
\sigma_{2}(G) & \geq|G|+b(H)-2,
\end{aligned}
$$

then $G$ is H-linked. This result is sharp. 
We also utilize Theorem 5 to give a sharp $\sigma_{2}$ bound that, without any additional minimum degree condition, assures a graph $G$ is $H$-linked for any simple graph $H$. Let

$$
a(H)=\max _{A \cup B=V(H)}\left(|E(A, B)|+|B|-\Delta_{B}(A)\right) .
$$

Theorem 6. Let $H$ be a simple graph and $G$ be a graph of order $n>20|E(H)|$. If

$$
\sigma_{2}(G) \geq n+a(H)-2
$$

then $G$ is H-linked. This result is sharp.

Observe that for arbitrary $H, a(H) \geq b(H)$. To see this, suppose that $V(H)=$ $A \cup B \cup C$ with $e(A, B)+|C|=b(H)$. Then, if we let $B^{*}=B \cup C$, it follows that

$$
a(H) \geq e\left(A, B^{*}\right)+\left|B^{*}\right|-\Delta_{B^{*}}(A) \geq e(A, B)+|C|=b(H) .
$$

There are a number of graphs $H$, including $C_{k}$, for which $a(H)>b(H)$. As such, Theorem 6 demonstrates that there are many choices of $H$ for which the $\sigma_{2}$-threshold for $H$-linkedness is more than twice the $\delta$-threshold.

\section{Preliminary Lemmas}

A version of the following Lemma originally appears in [12], pertaining to directed graphs. The proof for undirected graphs is analogous and, hence, omitted.

Lemma 7. Let $G$ be a graph, $k \geq 1$ and $v \in V(G)$ with $d(v) \geq 2 k-1$. If $G-v$ is $k$-linked, then $G$ is k-linked.

Thomas and Wollan [14] used the following to prove that every $10 \mathrm{~m}$-connected graph is $m$-linked, which represents the current best bound on connectivity sufficient to assure linkedness.

Theorem 8. Let $m \geq 2$ and $G$ be a $2 m$-connected graph. If $|E(G)| \geq 5 m|G|$, then $G$ is m-linked.

Corollary 9. Let $m \geq 2$ and $G$ be a $2 m$-connected graph of order $n$. If $\sigma_{2}(G) \geq n$ and $n \geq 20 m$, then $G$ is m-linked.

We close with the following straightforward fact and a useful, but equally straightforward, lemma.

Fact 10. Let $G$ be a graph and $H$ a (multi-)graph with $|E(H)|=m$ and $n_{0}(H)=0$. If $G$ is m-linked, then $G$ is H-linked.

Lemma 11. Let $H$ be a multigraph, and let $G$ be an edge maximal non- $H$-linked graph. Then for every $m \geq|E(H)|$ and $X \subseteq V(G)$ with $|X| \geq 2 m$ :

$$
G[X] \text { is } m \text {-linked } \Longleftrightarrow G[X] \text { is complete. }
$$




\section{Proofs of Theorems 5 And 6}

We are now ready to prove our main results.

Proof of Theorem 5. Sharpness is established by the following example, which is identical to the sharpness example for Theorem 3. Let $A \cup B \cup C$ be a partition of $V(H)$ such that $|E(A, B)|+|C|=b(H)$. Create $G$ by first adding $|E(A, B)|-1$ vertices to $C$ to obtain $C^{*}$, and then adding vertices to $A$ and $B$ to create sets $A^{*}$ and $B^{*}$, each of size $\frac{n-\left|C^{*}\right|}{2}$. The edges of $G$ are all possible edges in $\left(A^{*} \cup C^{*}\right)$ and $\left(B^{*} \cup C^{*}\right)$. It is straightforward to see that $G$ is not $H$-linked, as there is not a sufficient number of edges to create paths representing the edges in $E(A, B)$.

For the proof of the main statement of the Theorem, we will in fact show a slightly stronger statement as follows.

Claim 1. Let $H$ be a multigraph and $G$ be a graph with $|G| \geq 20|E(H)|+n_{0}(H)$, and let $V(H) \subseteq V(G)$. If

$$
\begin{aligned}
\delta(G) & \geq 4|E(H)|+n_{0}(H), \text { and } \\
d(x)+d(y) & \geq|G|+b(H)-2, \text { whenever } x, y \in V(G) \backslash V(H) \text { and } x y \notin E(G),
\end{aligned}
$$

then there is an H-linkage in $G$.

Let $n=|G|$ and $m=|E(H)|$. Note that the statement is trivial for $m \leq 1$, so we may also assume that $m \geq 2$.

For the sake of contradiction, we assume that there is no $H$-linkage in $G$, and furthermore that Claim 1 is true for every proper subgraph $H^{\prime} \subsetneq H$. Further, assume that $G$ is edge maximal without an $H$-linkage.

If $v \in V(H)$ is isolated in $H$, then solving the $H$-linkage problem in $G$ is equivalent to solving the associated $(H-v)$-linkage problem in $G-v$. As $G-v$ satisfies all of the conditions in Claim 1 (note that $b(H-v)=b(H)-1$ ), this yields a contradiction, so $H$ does not contain any isolated vertices.

If $G$ is $2 m$-connected, we are done by Corollary 9 , so we may assume that there is a minimal cut set $Z$ in $G$ with $|Z| \leq 2 m-1$. The degree conditions on $G$ imply that $G-Z$ has exactly two components, call them $X$ and $Y$ and we assume, without loss of generality, that $|X| \leq|Y|$. Let $x \in X$ and $y \in Y$, then

$$
n+b(H)-2 \leq d(x)+d(y) \leq|X|+|Y|+2|Z|-2 \leq n+|Z|-2,
$$

So

$$
\delta_{X}(X)+\delta_{Y}(Y) \geq|X|+|Y|-|Z|+b(H)-2 .
$$

Therefore,

$$
\delta_{X}(X) \geq \max \{|X|-|Z|+b(H)-1, \delta(G)-|Z|\} \geq|X|-\frac{3}{2} m .
$$

We now wish to show that both $X$ and $Y$ are $m$-linked. If $|X| \geq 5 m$, then $\delta_{X}(X) \geq \frac{7|X|}{2}$, so $X$ is $m$-linked by Theorem 1. Suppose, then, that $|X|<5 m$, so $2(|X|+|Z|)<|G|$ and $X$ is complete by the degree sum condition. Since $|X| \geq$ 
$\delta(G)+1-|Z| \geq 2 m+2$, the fact that $X$ is complete implies that $X$ is $m$-linked. Analogously, we also conclude that $Y$ is $m$-linked.

Let $z \in Z$, and suppose there are vertices $x \in X$ and $y \in Y$ such that $x z, y z \notin$ $E(G)$. Then

$$
n+|Z|+2 d(z) \geq d(x)+2 d(z)+d(y) \geq 2 n+m-4
$$

so

$$
d(z) \geq \frac{1}{2}(n+m-|Z|-4) \geq \frac{1}{2}(n-m-4)>6 m
$$

Thus, for every $z \in Z$, we have $d_{X}(z) \geq 2 m$ or $d_{Y}(z) \geq 2 m$. Let

$$
\begin{aligned}
B & :=\left\{v \in V(G): d_{Y}(v) \geq 2 m-1\right\}, \text { and } \\
A & :=V(G) \backslash B .
\end{aligned}
$$

Then, $A \supseteq X$ and $B \supseteq Y$ are $m$-linked by Lemma 7 , and therefore complete by Lemma 11. Let $A^{H}, B^{H}$ be the partition of $V(H)$ induced by this partition of $V(G)$.

Choose $a b \in E(H)$, let $H^{\prime}=H-a b$ and let $F \subseteq G$ be a solution of the $H^{\prime}$ linkage problem of minimum order. In particular, this implies that $|F \cap A| \leq 2 \mathrm{~m}$ and $|F \cap B| \leq 2 m$, so $A \backslash F \neq \emptyset$ and $B \backslash F \neq \emptyset$. Since $A$ and $B$ are complete, we conclude that $a \in A$ and $b \in B$, and in particular, $E(H)=E_{H}(A, B)$. By the minimality of $F$ we have $\left|E_{F}(A, B)\right|=\left|E_{H^{\prime}}(A, B)\right|=|E(H)|-1$.

Let $v \in A \backslash F$ and $w \in B \backslash F$. If $v w \in E(G)$, then we can extend $F$ to a solution of the $H$-linkage problem using the path avwb, so we conclude that $v w \notin E(G)$. Similarly, if there exists an $x \in(N(v) \cap N(w)) \backslash F$, we can extend $F$ to a solution of the $H$-linkage problem using avxwb, so $N(v) \cap N(w) \subseteq F$.

It is our goal to show that $|N(v) \cap N(w)| \leq\left|E_{F}(A, B)\right|$. Consider first $x y \in$ $E(F) \backslash E(H)$ with $x \in A$ and $y \in B$. If $x \in N(w)$ and $y \in N(v)$, then we can replace $x y$ by $x w$ and $v y$ in $F$ and solve the $H$-linkage problem, using one of the new edges instead of $x y$ and the other to connect $a$ and $b$. So $|N(v) \cap N(w) \cap\{x, y\}| \leq 1$ for all $x y \in E_{F}(A, B) \backslash E(H)$.

Now, let $x y \in E(F) \cap E(H)$ with $x \in A$ and $y \in B$. If $\{x, y\}=\{a, b\}$ (so that $a$ and $b$ are joined by at least two edges in $H)$, then by the same argument as above, $|N(v) \cap N(w) \cap\{x, y\}| \leq 1$. If, instead $\{x, y\} \neq\{a, b\}$, then there is another edge $x^{\prime} y^{\prime} \in E(F)$ with $x^{\prime} \in A$ and $y^{\prime} \in B$ that lies on an $x-y$ path in $F$. Now, if $x=x^{\prime}$ (or, nearly identically, if $y=y^{\prime}$ ) then as above, $\left|N(v) \cap N(w) \cap\left\{x, y^{\prime}\right\}\right| \leq 1$, and so $\left|N(v) \cap N(w) \cap\left\{x, y, x^{\prime}, y^{\prime}\right\}\right| \leq 2$. Also, if $x \neq x^{\prime}, y \neq y^{\prime}$, and $v y, x^{\prime} w \in E(\bar{G})$, then we can replace $x^{\prime} y^{\prime}$ by $v y$ in $F$ and use $x^{\prime} w$ to connect $a$ and $b$. Similarly, we can't have $x \neq x^{\prime}, y \neq y^{\prime}$ and both of $x w, v y^{\prime} \in E(G)$, so again, $\left|N(v) \cap N(w) \cap\left\{x, y, x^{\prime}, y^{\prime}\right\}\right| \leq 2$.

We therefore conclude that $|N(v) \cap N(w)| \leq\left|E_{F}(A, B)\right|$. This yields a contradiction, as then

$$
a(H) \leq|N(v) \cap N(w)| \leq\left|E_{F}(A, B)\right|=|E(H)|-1 \leq a(H)-1 .
$$


Proof of Theorem 6. Sharpness follows from the following example. Starting from a partition $A \cup B$ of $V(H)$ with $\left(|E(A, B)|+|B|-\Delta_{B}(A)\right)=a(H)$, add a set $C$ of $|E(A, B)|-1$ vertices. Blow up $B$ to $B^{*}$ by adding $n-|A|-|B|-|C|$ vertices to $B$ and then add all edges in $A \cup C, B^{*} \cup C$, and all edges between $A$ and $B$ except for the edges in $H$. This graph is not $H$-linked, as there is not a sufficient number of vertices in $C$ to create paths representing the edges in $E(A, B)$, and has $\sigma_{2}=n+a(H)-3$.

As in the proof of Theorem 5, we may assume that $n_{0}(H)=0$ as isolated vertices in $H$ contribute 2 to $|G|+a(H)$ and at most 2 to $\sigma_{2}(G)$.

For the sake of contradiction, we assume that $G$ is not $H$-linked, and furthermore that $G$ is edge maximal with this property. Let $m=|E(H)|$ and $n=|G|$.

If $\delta(G) \geq 4 m$, we are done by Theorem 5 (as $b(H) \leq a(H)$ ), so there is a vertex $v$ with $d(v)<4 m$. Let $Y:=V(G) \backslash N[v]$. Then $|Y|>16 m$ and

$$
\delta_{Y}(Y)>|Y|-4 m>\frac{1}{2}|Y|+m,
$$

and therefore $Y$ is $m$-linked by Theorem 1 . Let $B \supseteq Y$ be maximal such that $B$ is $m$-linked, and $A:=V(G) \backslash B \subseteq N[v]$. If $A=\emptyset$, we are done so we assume that $A \neq \emptyset$. By Lemma 7 no vertex in $A$ has $2 m$ neighbors in $B$, so $\Delta_{G}(A)<6 m$ and therefore $A$ is complete by the degree sum condition. We now continue in a manner similar to the proof of Theorem 5 .

We may assume that $G$ is $H^{\prime}$-linked for every proper subgraph $H^{\prime} \subsetneq H$, as otherwise we could continue with a minimal subgraph $H^{\prime}$ of $H$ for which $G$ is not $H^{\prime}$-linked and observe that $a\left(H^{\prime}\right) \leq a(H)$. Let $A^{H} \cup B^{H}$ be the partition of $V(H)$ induced by $A$ and $B$. Note that $B$ is complete by Lemma 11. If there is an edge $e \in E(H) \cap E(G)$, we can extend any solution of the $(H-e)$-linkage problem trivially to a solution of the $H$-linkage problem, so we conclude that $E(H) \cap E(G)=\emptyset$, and in particular, $E(H)=E_{H}(A, B)$.

Let $a \in A^{H}$ maximize $\left|E_{H}(a, B)\right|$, and let $a b \in E(H)$. Let $H^{\prime}=H-a b$ and let $F \subseteq G$ be a solution of the $H^{\prime}$-linkage problem of minimum order, so in particular $\left|E_{F}(A, B)\right|=\left|E\left(H^{\prime}\right)\right|$.

Let $w \in B \backslash F$. If $a w \in E(G)$, then we can extend $F$ to a solution of the $H$-linkage problem using the path $a w b$, so we conclude that $a w \notin E(G)$. Similarly, if there exists an $x \in(N(a) \cap N(w)) \backslash F$, we can extend $F$ to a solution of the $H$-linkage problem using $a x w b$, so $N(a) \cap N(w) \subseteq F$. Now let $x y \in E(F)$ with $x \in A$ and $y \in B \backslash B^{H}$. If $x \in N(w)$ and $y \in N(a)$, then we can replace $x y$ by $x w$ and $a y$ in $F$ and solve the $H$-linkage problem. Thus, all edges $x y \in E(F)$ with $\{x, y\} \subset N(a) \cap N(w)$ have $y \in B^{H} \backslash N_{H}(a)$. But this yields a contradiction, as then

$$
\begin{aligned}
a(H) \leq|N(a) \cap N(w)| \leq|E(F)| & +\left|B^{H} \backslash N_{H}(a)\right| \\
& =|E(H)|-1+\left|B^{H}\right|-\Delta_{B^{H}}\left(A^{H}\right) \leq a(H)-1 .
\end{aligned}
$$


We note here that Theorem 6 does not extend to arbitrary multigraphs $H$. To see this, let $k \geq 6, r=2(k-1)$, and let $H$ be the disjoint union of a star having center $c$ and leaves $\ell_{1}, \ldots, \ell_{r}$ with an edge $u v$ of multiplicity $k$. As defined above, $a(H)=3 k-1$ (let $B$ consist of $u$ and all of the $\ell_{i}$ ). However, consider the following example. Let $A=\{c, u, v\}$ be a triangle and $X$ be a clique of order $n-3$ containing disjoint subsets $L, X_{u}$ and $X_{v}$ of $X$ with $\left|X_{v}\right|=r,\left|X_{u}\right|=r-1$ and $L=\left\{\ell_{1}, \ldots, \ell_{r}\right\}$.

Construct $G$ from $A$ and $X$ by adding all edges from $u$ to $X_{u} \cup L, v$ to $X_{v} \cup L$ and $c$ to $X_{u} \cup X_{v}$ and note that $\sigma_{2}(G)=n+(4 k-4)-2>n+a(H)-2$. If we let the vertex labels in $G$ define an $H$-linkage problem $\rho$, then we require at least one vertex from $X_{u} \cup X_{v}$ to construct the $r$ desired paths from $c$ to $L$ and at least two vertices from $X_{u} \cup X_{v}$ to construct each of the remaining $k-1$ paths from $u$ to $v$. This is a total of at least $2 k-4$ additional vertices, which exceeds the $2 k-5$ vertices in $X_{u} \cup X_{v}$. Hence $G$ is not $H$-linked.

Theorems 5 and 6 also allow us to obtain a number of interesting results on $k$-linked and $k$-ordered graphs as corollaries. In particular, we obtain the degree conditions for sufficiently large $k$-linked, $k$-ordered and $H$-linked graphs found in Theorems 2,3 and 4 , respectively. In most cases, our bounds on $|G|$ are reasonable, but slightly larger than those in the original theorems due to the more general nature of our results.

\section{REFERENCES}

1. R. Diestel, "Graph Theory," Springer Verlag, Heidelberg, 2005.

2. J. Faudree, R. Faudree, R. Gould, M. Jacobson and L. Lesniak, On k-ordered graphs, J. Graph Theory 35 (2000), 69-82.

3. M. Ferrara, R.J. Gould, G. Tansey and T. Whalen, On H-linked graphs, Graphs. Combin. 22 (2006), 217-224.

4. M. Ferrara, R.J. Gould, G. Tansey and T. Whalen, On H-immersions, J. Graph Theory 57 (2008), 245-254.

5. R. Gould, A. Kostochka, G. Yu, On minimum degree implying that a graph is $H$-linked, $S I A M$ J. Discrete Math. 20 (2006), 829-840.

6. R. Gould and T. Whalen, Distance between two $k$-sets and path-systems extendibility, Ars Combin. 79 (2006), 211-228.

7. H. A. Jung, Eine verallgemeinrung des $n$-fachen zusammenhangs fur graphen, Math. Ann. 187 (1970), 95-103.

8. K. Karawabayashi, A. Kostochka and G. Yu, On sufficient degree conditions for a graph to be $k$-linked, Combin. Probab. Comput. 15 (2006), 685-694.

9. H. Kierstead, G. Sárközy and S. Selkow, On k-ordered Hamiltonian graphs, J. Graph Theory 32, 17-25.

10. A. Kostochka and G. Yu, Minimum degree conditions for H-linked graphs, Discrete Appl. Math. 156 (2008), 1542-1548.

11. A. Kostochka and G. Yu, Ore-type degree conditions for $H$-linked graphs, J. Graph Theory 58 (2008), 14-26.

12. Y. Manoussakis, $k$-linked and $k$-cyclic digraphs, J. Comb. Theory Ser. B 48 (1990), 216-226.

13. O. Ore, A Note on Hamilton Circuits, Amer. Math. Monthly 67 (1960), 55.

14. R. Thomas and P. Wollan, An improved linear edge bound for graph linkage. European J. Combin. 26 (2005), 309-324. 